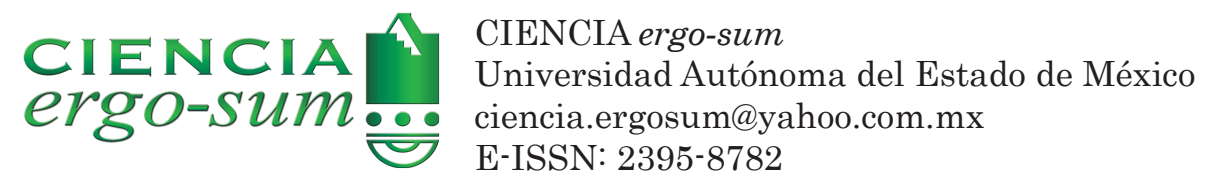

\title{
Cambio climático. Percepciones sobre manifestaciones, causas e impactos en el Distrito de Temporal Tecnificado Margaritas-Comitán, Chiapas
}

\author{
Soares, Denise; García, Antonino; Manzano Solís, Luis Ricardo \\ Margaritas-Comitán, Chiapas \\ CIENCIA ergo-sum, vol. 25, núm. 1, 2018|e1 \\ Universidad Autónoma del Estado de México, México
}

Cambio climático. Percepciones sobre manifestaciones, causas e impactos en el Distrito de Temporal Tecnificado

Soares, D., García, A. y Manzano Solís, L. R. (2018). Cambio climático. Percepciones sobre manifestaciones, causas e impactos en el Distrito de Temporal Tecnificado Margaritas-Comitán, Chiapas. CIENCIA ergo-sum, 25(1).

https://doi.org/10.30878/ces.v25n1a1 


\section{Cambio climático. Percepciones sobre manifestaciones, causas e impactos en el Distrito de Temporal Tecnificado Margaritas-Comitán, Chiapas}

Climate change. Perceptions of manifestations, causes and impacts in the Technified Rainfed District of MargaritasComitán, Chiapas

Denise Soares

Instituto Mexicano de Tecnología del Agua, México

Recepción: 10 de noviembre de 2016

denisefsoares@yahoo.com.mx

Antonino García

Universidad Autónoma Chapingo-sede Chipas, México

tonygg@prodigy.net.mx

Luis Ricardo Manzano Solís

Universidad Autónoma del Estado de México, México

luisrms@gmail.com

\section{RESUMEN:}

Se exploran percepciones acerca de las manifestaciones del cambio climático, sus causas e impactos en el cotidiano de actores sociales locales en el Distrito de Temporal Tecnificado Margaritas-Comitán (DTT011) ubicado en la Meseta Comiteca, estado de Chiapas, perteneciente a la parte alta de la cuenca del río Usumacinta. Asimismo, se propone argumentar con base en información empírica que, independiente de la aprehensión del concepto de cambio climático, a nivel local se observa, interpreta y siente el impacto de las variaciones en los periodos de lluvia y del incremento del calor, las cuales presentan consecuencias en términos económicos en la zona, toda vez que los sistemas productivos se ven afectados y se pierden cosechas.

Palabras ClaVE: cambio climático, sistemas productivos, percepciones, vulnerabilidad.

\section{Abstract:}

This article explores perceptions about climate change manifestations, their causes and impacts on the daily lives of local social actors in the Technified Rainfed District of Margaritas Comitán, Chiapas (DTT011) located in Chiapas State, in the upper part of the Usumacinta River Basin. This research proposes to argue, based on empirical information, that irrespective of the perception of the concept of climate change, the impact of the variations in the periods of rain and the increase of the heat are observed, interpreted and felt at local level and these variations are having economic consequences, in that way, production systems are affected and crops are lost.

KEYWORDS: climate change, productive systems, perceptions, vulnerability.

\section{INTRODUCCIÓN}

El cambio climático es evidente en todo el planeta. Sus impactos adversos sobre la población, los recursos naturales y la economía constituyen actualmente uno de los problemas más apremiantes para la mayoría de los países. Desde siempre la humanidad ha enfrentado los efectos del clima como parte de los diversos fenómenos naturales que ha de experimentar; sin embargo, la agudización de algunas manifestaciones climáticas como la intensidad y frecuencia de tormentas y huracanes, la abundancia de las precipitaciones pluviales con las consecuentes inundaciones, deslizamientos, desbordamiento de ríos y presas, las olas de calor y las sequías de duración inusual son muestras de que las variaciones del clima van más allá de lo que se considera natural.

Como lo plantean Giddens y Sutton (2013), el tema ha pasado para el centro del debate público como el mayor desafío del siglo XXI. Entre los factores que contribuyeron para elevar la conciencia pública sobre la gravedad de la situación que pueden plantear el cambio climático se encuentran por ejemplo el Informe de 2007 del Panel Intergubernamental sobre el Cambio Climático (IPCC, 2008) y también la película U na 
verdad inómoda (2006) de Al Gore. Además, no se puede menospreciar el papel que han jugado diversos desastres, entre ellos el huracán Katrina en 2005, que dejó en ruina la ciudad de Nueva Orleans, ubicada en el país más poderoso del mundo, y una ola de calor en 2003, que ocasionó la muerte de alrededor de 30 mil personas en Europa.

El cambio climático agravará los riesgos existentes y creará nuevos para los sistemas naturales y humanos. Los riesgos se distribuyen de forma dispar y son en general mayores para las personas y comunidades más desfavorecidas de los diferentes países. Los impactos en los sistemas humanos suelen ser heterogéneos geográficamente porque dependen no sólo de cambios en las variables climáticas, sino también de factores sociales y económicos. De esa manera, se pone de manifiesto la vulnerabilidad social, dado que los desastres están asociados en gran medida a esto; por ello, serán los países que producen menor cantidad de emisiones los que tendrán sus sistemas naturales y humanos más afectados debido a sus medios de sustento menos seguros, su dependencia de recursos naturales, su marginación y mayor vulnerabilidad al hambre y a la pobreza, entre otros (PNUD, 2008; IPCC, 2014).

No obstante el carácter antropogénico del debate sobre el cambio climático, dado que en el centro de la discusión están las actividades humanas que generan emisiones crecientes de $\mathrm{CO}_{2}, \mathrm{CH}_{4}$ y otros gases de efecto invernadero, las ciencias naturales y exactas vienen dominando el debate científico en la materia y son relativamente recientes los aportes de las ciencias sociales, los cuales están dedicados sobre todo a la comprensión de las actividades humanas que provocan el cambio climático, así como a diagnosticar la vulnerabilidad de distintos grupos sociales y sectores a las variaciones climáticas y a analizar las percepciones sociales frente a dichas amenazas (Hogan, 2007).

Las ciencias exactas y naturales hacen aproximaciones al cambio climático a través del abordaje de las transformaciones en la atmósfera y su interacción a diversas escalas con el mar y el continente. La climatología determina que el cambio climático es el resultado de los gases invernadero que el ser humano ha esparcido en la atmósfera terrestre a partir de la industrialización, en donde el dióxido de carbono es el gas predominante, producto de la quema de fuentes de energías fósiles. Por otro lado, para los estudiosos de las ciencias sociales el concepto de clima es una construcción cultural, elaborada a partir de procesos materiales y simbólicos, de tal suerte que la conceptualización deriva de cómo las personas perciben, se apropian, interpretan y padecen los eventos meteorológicos y climáticos que ocurren a su alrededor. De este modo, cuando se dejan de lado las dimensiones físicas del fenómeno, como la única manifestación del cambio climático y se baja a una escala más local, se puede constatar que cada cultura tiene su propia percepción del cambio climático, que las reacciones humanas son variadas y que están arraigadas en entornos culturales y contextos político-sociales específicos (Rossbach de Olmos, 2011; Mariño, 2011; Soares y Romero, 2008).

Según Kuhnem e Higuchi (2011), la percepción ambiental se refiere a la forma como las personas se relacionan con el medioambiente y está integrada por aspectos materiales, psicosociales, históricos y socioculturales. Para Marson Teixeira de Andrade y Miccolis (2012) la percepción está fundamentalmente relacionada con el contexto en el cual se vive, de tal suerte que al experimentar el riesgo en lo cotidiano se conformará la percepción del mismo. Slovic (1987) abona a dicho planteamiento al argumentar que existen factores cualitativos subyacentes a las percepciones de riesgo, los cuales son movilizados mentalmente para la evaluación del riesgo.

La relevancia de estudiar las percepciones radica en el hecho de que la adaptación al cambio climático será asumida por las personas a nivel local en la medida en que perciban dicho fenómeno (Maddison, 2007). En este mismo orden, Tuan (1980) distingue que la autocomprensión es fundamental para encontrar soluciones para los desafíos ambientales y desde luego el cambio climático es uno de los mayores desafíos ambientales que la humanidad está enfrentando en el siglo XXI. Whyte (1977) propone que este abordaje investigativo contribuye para un uso y manejo sustentable de los recursos por favorecer la participación social en la planeación y el desarrollo local. Pinilla-Herrera et al. (2012) añaden que el conocimiento local representa un 
importante elemento para comprender los mecanismos de adaptación ya adoptados tradicionalmente por los agricultores en su búsqueda de alternativas para convivir con el cambio climático.

Un acercamiento a las comunidades agrícolas y campesinas desde los postulados de las ciencias naturales (atmosféricas y ambientales), así como de las ciencias sociales se circunscribe dentro de una plataforma etic, es decir, de la construcción social (imaginario externo) del fenómeno cambio climático con marcos conceptuales de la ciencia occidental. La percepción de cada uno de los miembros de una familia o comunidad - plataforma emic- será diferente a la externa en tanto sujetos de una cultura construida y apropiada en escalas comunitarias y regionales, con conocimientos empíricos del fenómeno de acuerdo con lo observado, sentido y procesado en lo mental y sentimental.

Para esta investigación, la diferencia de fondo entre el misionero-lingüista Kenneth Lee Pike (1912-2000), que acuña los conceptos emic y etic, con el antropólogo Marvin Harris (1927-2001) no es tan distante como lo han planteado diferentes acercamientos antropológicos (Harris, 1982; Pike y Pike, 1991). El primero pretende moverse dentro de la esencia del templo cultural de los pueblos y comunidades buscando las subjetividades, valores y juicios de los actores (emic), mientras que el segundo se mueve en el materialismo cultural, es decir, en una ciencia antropológica de corte positivista que pretende ser objetiva a través de descripciones etic (el análisis del antropólogo científico como agente externo). En el caso de este trabajo ambos acercamientos se complementan en tanto que las prácticas culturales agrícolas para sortear las variaciones de los periodos de lluvia y el incremento del calor se hacen con el conocimiento empírico de los campesinos, independientemente de la evidencia externa de las plataformas internacionales del fenómeno cambio climático. Así, un método heurístico de análisis deberá considerar tanto el conocimiento externo (etic) como el conocimiento interno (emic) sobre el fenómeno cambio climático. Esta visión integradora permitirá la hechura, ejecución y evaluación de políticas públicas pertinentes para abordar el principal fenómeno global que enfrenta hoy en día la humanidad.

Este artículo, concebido desde el referente de las ciencias sociales, trata de abonar a la discusión sobre cómo el cambio climático (construcción etic) es percibido a nivel local (construcción emic) a partir de los hallazgos encontrados en el trabajo de campo realizado en el Distrito de Temporal Tecnificado Margaritas-Comitán (DTT011), ubicado en el estado de Chiapas. A través de técnicas cualitativas de investigación, el estudio busca contestar a las siguientes interrogantes: ¿Cuáles son las manifestaciones del cambio climático a nivel local?, ¿cuáles son sus causas desde la perspectiva de actores locales? y ¿qué impactos tiene el cambio climático en el cotidiano de las personas?

\section{1. Área de ESTUdio}

El Distrito de Temporal Tecnificado Margaritas-Comitán (DTT011) comprende un área geográfica de 47 997.13 ha, localizada en los municipios de Comitán de Domínguez, La Independencia, Las Margaritas y La Trinitaria. Esta área se encuentra dentro de la región conocida como Meseta Comiteca Tojolabal en el estado de Chiapas. ${ }^{[1]}$ Geográficamente, el DTT011 se localiza entre las coordenadas 91 58' 08.3” de longitud oeste y $16^{\circ} 15^{\prime} 38.5^{\prime \prime}$ de latitud norte. En cuanto a la hidrología el área comprendida por el DTT011 se localiza en la gran cuenca del río Usumacinta, y una parte de esta área pertenece a la subcuenca río Grande de Comitán-Lagunas de Montebello. Cabe señalar que la Comisión Nacional para el Conocimiento y Uso de la Biodiversidad (CONABIO) identifica a esta subcuenca como una región hidrológica prioritaria y fue incluida dentro de un proyecto de diagnóstico para las principales subcuencas y sistemas acuáticos del país susceptibles de ser integradas a programas de manejo y conservación (García García et al., 2015).

La justificación para estudiar las comunidades dentro del DTT011, pertenecientes al municipio de La Independencia, Chiapas, se debe a que se encuentran en el corazón de la histórica región agrícola Meseta Comiteca que ha sufrido, como ninguna otra región del estado, el impacto del cambio climático al disminuir de dos ciclos agrícolas de temporal a uno. Debido a lo anterior, la población buscó alternativas diversificando 
la producción hacia hortalizas, principalmente tomate. Las comunidades en donde se realizó el trabajo de campo fueron pioneras en la introducción del cultivo de tomate, la perforación de pozos profundos para mitigar el ciclo irregular de precipitación, así como en la conformación de unidades de riego dentro del DTT011. En este sentido, el análisis hecho se puede utilizar para delinear acciones tendientes a la mitigación y adaptación al cambio climático por parte de los tres niveles de gobierno, organismos no gubernamentales y sociedad civil de la zona de estudio.

En términos de población el DTT011 cuenta con un total de 39050 habitantes distribuidos en 150 localidades pertenecientes a los cuatro municipios en los que se localiza el distrito. La mayor parte de la población del DTT011 se encuentra asentada en las comunidades que pertenecen al municipio de La Independencia (46.7\%), una razón más para trabajar en comunidades de ese municipio. Históricamente las comunidades del DTT011 han incrementado su población debido a dos factores: a) las superficies de tierra manejadas por familia eran considerables (promedio $10 \mathrm{ha}$ ) y la calidad de los suelos fue alta en su momento y $b$ ) era la única región del estado de Chiapas donde todavía hace 25 años se cosechaban dos ciclos de milpa de temporal. Desafortunadamente esa productividad en granos básicos se fue perdiendo a raíz de la introducción del cultivo de tomate en los últimos 20 años y a la variabilidad de los ciclos de lluvia. Hoy en día el calentamiento global se deja sentir en la Meseta Comiteca y su impacto es observable vía registro de siembras siniestradas por sequía en los últimos 10 años (García García et al., 2015).

En relación con la climatología, el rango de temperaturas en el DTT011 presenta pocas variaciones. Se estima que la temperatura máxima promedio es de $25.5^{\circ} \mathrm{C}$ durante el periodo de mayo a octubre, mientras que el rango de variación durante este periodo va de los $24^{\circ} \mathrm{C}$ a los $27^{\circ} \mathrm{C}$. En cuanto a la precipitación, en el área de influencia del DTT011 las lluvias se presentan en su mayoría en verano. El rango de precipitación que prevalece en la mayor parte de la zona para el periodo de mayo a octubre, va de 900 a $1000 \mathrm{~mm}$, el cual se presenta en $64 \%$ de su territorio en tanto que los niveles que van de 1000 a $1200 \mathrm{~mm}$ se manifiestan en 24.2\% del territorio del distrito. Durante el periodo de noviembre a abril, los niveles de lluvia disminuyen de manera significativa, por lo que esta época es considerada como la estación seca. Los niveles de precipitación cuantificados en este periodo presentan rangos máximos que van de los 350 a los $400 \mathrm{~mm}$ en una porción del territorio del distrito de temporal que equivale a 2.5\% respecto del total (García García et al., 2015).

García García et al. (2015) señalan que, según información de los productores agropecuarios ubicados dentro del distrito, en los últimos 10 años existen evidencias claras de que ha disminuido la precipitación en los periodos de lluvia provocando incluso pérdida de siembras de milpa debido a las alteraciones en los ciclos de lluvia; también mencionan que la variabilidad del ciclo de lluvia es una constante, es decir, periodos cortos (tres a cinco días) con lluvias extremas y periodos largos (una a seis semanas) donde no llueve.

De las actividades económicas primarias que se realizan en el área de influencia del DTT011, la agricultura es la que más se practica y es de gran importancia para las comunidades rurales, ya que sirve como base para el sustento familiar. Sin embargo, existe una diferencia entre los cuatro municipios que integran el área en relación con los sectores de ocupación, tal como se muestra en el cuadro 1.

CUADRO 1

Porcentaje de la población económicamente activa ocupada por sector a nivel municipal.

\begin{tabular}{|lccc}
\hline \multicolumn{1}{c}{ Municipio } & Primario & Secundario & Terciario \\
\hline Comitán de Domínguez & $16 \%$ & $21 \%$ & $63 \%$ \\
La Independencia & $86 \%$ & $4 \%$ & $9 \%$ \\
Las Margaritas & $82 \%$ & $6 \%$ & $11 \%$ \\
La Trinitaria & $77 \%$ & $9 \%$ & 13 \\
\hline
\end{tabular}

Fuente: INEGI (2011). 
En el cuadro 1 se observa que, en cuanto a la ocupación del sector primario, es el municipio de La Independencia el que mantiene un mayor porcentaje de su población económicamente activa ocupada con 86\%; le siguen los municipios de Las Margaritas con 82\%, La Trinitaria con 77\% y Comitán de Domínguez con $16 \%$. Cabe señalar que, a pesar de que la agricultura milpera ha sido históricamente la principal actividad que realizan los habitantes del DTT011, en los últimos años se ha observado un decremento de la misma. Por un lado, este fenómeno se debe a la adopción de nuevos cultivos como el tomate y otras hortalizas que se cotizan a mayor precio en el mercado, pero por el otro se relaciona con los problemas asociados con el uso indiscriminado de los agroquímicos ${ }^{[2]}$ y los fuertes procesos de deforestación que han deteriorado la calidad de los suelos del DTT011 (García García et al., 2015). La irregularidad en los periodos de las lluvias también se viene conformando como una de las causas para el abandono paulatino de la actividad milpera.

Los cultivos de maíz y frijol representan la mayor cantidad de la superficie agrícola en el DTT011; sin embargo, existen otros cultivos como el tomate y las hortalizas que a pesar de no ser representativos a nivel de superficie son importantes para los productores porque generan un relevante ingreso económico. En el área se identificaron dos ciclos para los diversos sistemas de producción que se desarrollan: primavera-verano y otoño-invierno.

La baja productividad agrícola ha provocado que algunos productores emigren en busca de fuentes de empleo a ciudades como Cancún, Puerto Vallarta, Playa del Carmen e Isla Mujeres, lo que se traduce en un abandono de las parcelas. Se estima que alrededor de $25 \%$ de los jóvenes entre 18 y 28 años va en busca de trabajo a los Estados Unidos de América (García García et al., 2015).

Una de las principales problemáticas en el área es el uso intensivo de insumos para la siembra de tomate. Este cultivo requiere el uso de grandes cantidades de agroquímicos para el control de plagas y enfermedades. Finalmente, los sistemas de producción son afectados por otros factores que limitan su desarrollo y rentabilidad, entre ellos la descapitalización y la poca organización de los productores, la falta de capacitación y de asesoría técnica, los bajos precios y la falta de mercado, el cual lleva a que existan intermediarios para la compra de los productos (García García et al., 2015). Un factor reciente que se suma a la problemática vivida por los agricultores en la región es la irregularidad en el periodo de lluvias y las sequías recurrentes, los cuales impactan de manera decisiva en la producción agrícola.

\section{Metodología}

Para el desarrollo de este estudio se revisó bibliografía sobre los temas de cambio climático y percepciones. Enseguida se llevó a cabo el trabajo de campo para generar información empírica con la aplicación de entrevistas como herramientas básicas para obtener información primaria relativa a percepciones sociales sobre los impactos del cambio climático en la región del DTT011. Dicho estudio, de corte cualitativo, no pretende brindar información que pueda ser generalizada como las percepciones imperantes en la zona de estudio, sino que es un acercamiento a un determinado grupo de personas, compuesto por campesinos plantadores de tomate, asalariados del sector comercio y de una empresa perforadora de pozos y funcionarios públicos.

El diseño de las entrevistas contempló definir las variables relacionadas con la identificación de las personas informantes y su percepción relativa a las manifestaciones del cambio climático, sus causas e impactos en el cotidiano de las personas entrevistadas. Se aplicaron 18 entrevistas semiestructuradas a 38 informantes clave. Una de las entrevistas fue colectiva, en la que participaron 20 campesinos pertenecientes a una asociación de productores de tomate. Entre las personas entrevistadas se encuentran funcionarios del municipio de La Independencia (director de protección civil, subdirector agropecuario, director del sistema de agua), de la Conagua (jefe del DTT011) y de la Secretaría de Educación Pública (SEP) (Brigada de educación para 
el desarrollo rural en la Meseta Comiteca). Nos ha parecido relevante incluirlas en el estudio por su papel como representantes de instituciones que deberían estar jugando un rol decisivo en el desarrollo de políticas públicas de cambio climático a nivel local.

\section{EL CAMBIO CLIMÁTICO SEGÚN LA PERCEPCIÓN DE LOS ACTORES SOCIALES LOCALES}

\section{1. Manifestaciones del cambio climático}

Los datos empíricos obtenidos a través de entrevistas permiten explorar la percepción de los pobladores del DTT011, quienes en su mayoría no poseen un discurso elaborado sobre el cambio climático; por el contrario, aseveran no haber oído hablar del fenómeno. Frente a la situación de desconocimiento del concepto por muchos de los actores sociales locales, se optó por emplear una estrategia de acercamiento al concepto de cambio climático de manera indirecta en el que se hace mención a sus manifestaciones. Así se realizó un abordaje a través de los siguientes componentes: $a$ ) irregularidad en la distribución de las lluvias, $b$ ) cambio en el comportamiento de las granizadas y $c$ ) incremento de la temperatura.

Los resultados de aplicar esa estrategia reflejan una preocupación significativa de los individuos frente al fenómeno por reconocer que el clima está cambiando, pues las precipitaciones han modificado de manera significativa sus patrones, a punto de ser impredecible la entrada de la temporada de lluvias. Por ejemplo, las granizadas caen en periodos no esperados y es perceptible que la temperatura está aumentando y trae consecuencias sociales y ambientales. En cuanto a la definición temporal acerca del inicio de los cambios mencionados no hay un consenso entre las personas entrevistadas, sino que se rastrea el inicio de estos cambios en el clima entre 30, 20 o 10 años atrás. A continuación, algunos testimonios respecto a lo expuesto:

Hace ocho o diez años empezó el problema del descontrol de las lluvias. Anteriormente las granizadas caían con los primeros aguaceros, en mayo. Y era una bendición para las milpas, pues mataba a las plagas. Ahora el granizo cae diferente, ya no sabemos cuándo va a caer y ya no ayuda. El calor que nos pegaba anteriormente era diferente. Antes era más leve, el de ahorita se siente muy quemante. Antes daba gusto recibir este calor, ahora no; es más quemante y aquí es clima templado (testimonio entrevistado 1).

La lluvia se viene alejando y el calor se siente más fuerte. Ahora la lluvia cae muy fuerte y termina pronto. Antes tardaba más en caer. Hace 20 años empezó a cambiar (testimonio entrevista colectiva 11).

La totalidad de los campesinos entrevistados no conocía el concepto de cambio climático, pero sí observaba cambios en la región de acuerdo con los testimonios. Cuatro personas asalariadas (tres mujeres trabajadoras de una paletería en El Triunfo y un hombre empleado de una empresa perforadora de pozos en Buenavista, ambas localidades pertenecientes al municipio La Independencia) manifestaron conocer el concepto de cambio climático. Por una parte, las mujeres lo definieron como un daño que los seres humanos están provocando a la capa de ozono, aunado al exceso en el uso de agroquímicos en la agricultura; por otra, el hombre lo asoció directamente a la irregularidad de las lluvias y de las granizadas y al incremento de la temperatura y del calor.

La percepción que asocia el cambio climático a la capa de ozono está vinculada a los reportes que transmiten por los medios de comunicación, en tanto que el uso de agroquímicos y la irregularidad de las lluvias y granizadas pareciera estar asociada a una vivencia personal y local. Asimismo, la percepción asociada al incremento de la temperatura y del calor del sol coincide con VanderMolen (2011), quien ha encontrado en su investigación de comunidades campesinas en Ecuador que un porcentaje de las personas entrevistadas percibe que la temperatura ha aumentado y que el sol está más fuerte que antes. Siguiendo esta línea, Paes de Menezes et al. (2011) han encontrado lo mismo en su investigación sobre impresiones acerca del cambio climático con campesinos en la región semiárida brasileña, es decir, existe una percepción generalizada a nivel local acerca del incremento de la temperatura y de la sensación de calor, que coincide con la ciencia occidental 
positivista al registrar un incremento de temperatura gradual y que según las predicciones llegará a un pico insalvable en 2100 .

La percepción de los campesinos de México, Ecuador y Brasil coinciden con el planteamiento del Cuarto Informe de Evaluación del Grupo Intergubernamental de Expertos sobre el Cambio Climático, el cual asevera que el cambio climático provocará un incremento medio global de la temperatura del planeta entre $1.8^{\circ} \mathrm{C}$ y $4.0^{\circ} \mathrm{C}$ hasta 2100 . Añade el informe que dicho incremento puede alcanzar los $6.4^{\circ} \mathrm{C}$ si la población y la economía mundial mantienen la misma lógica de crecimiento, con un consumo intensivo de combustibles fósiles. De igual modo, plantea que las comunidades pobres son las que más sufrirán los efectos del cambio climático por tener una capacidad de adaptación limitada y ser más dependientes de los recursos naturales (IPCC, 2008).

\section{2. Causas del cambio climático}

A diferencia de las percepciones sobre las manifestaciones del cambio climático, en donde hay una confluencia entre las respuestas hacia las lluvias, granizadas y las temperaturas, al preguntar sobre sus causas podemos clasificar las respuestas en tres grandes temas: a) el primero asociado a cuestiones místicas, en donde Dios es el que lo envía como castigo, $b$ ) el segundo relacionado con el ciclo hidrológico, en donde la deforestación hace que las lluvias se "alejen" y c) el tercero en donde la contaminación de los suelos por los agroquímicos es la gran culpable. En cuanto a la interpretación mística las personas nada pueden hacer para cambiar el rumbo de las cosas, pues es fruto de un castigo divino. En la segunda y la tercera interpretación se asumen como los causantes del cambio climático y como tal vislumbran que también está en sus manos parte de la solución.

Retomamos, a manera de ejemplo, un testimonio que plantea las causas del cambio climático como voluntad divina:

Dios está muy molesto con los hombres porque hemos pecado y sus palabras son éstas: más calor, menos lluvias y nuevas enfermedades para que entendamos que hay que tener temor a Dios y arrepentir de nuestros pecados (testimonio entrevistado 18).

Desde esta racionalidad, no se hace necesario que las poblaciones y gobiernos inviertan energía y recursos para promover la adaptación al cambio climático, dado que ello se deriva de la relación entre los seres humanos con su Dios y no de la articulación de las poblaciones con la naturaleza. Desde esta perspectiva, la adaptación mediante estrategias alternativas de producción y consumo cede paso a la "salvación" a través del arrepentimiento y la autocorrección (Soares y García, 2014). Según Flores Moreno y Valdivia Corrales (2011), este discurso ofrece un grado compensatorio de certidumbre en un marco de incertidumbres globales que cuestionan las perspectivas de riesgos conocidas y acrecientan el conflicto cultural.

Relacionado con la interpretación que asocia la causa del cambio climático a la deforestación, el hecho de que los suelos no tengan suficiente cobertura forestal provoca que se reduzca el proceso de evapotranspiración y con ello la formación de las nubes, lo cual provoca que las lluvias se "alejen", según comentarios de los actores sociales locales. Es evidente la gran dependencia de la agricultura de temporal hacia las lluvias, por lo cual la reducción de las precipitaciones resulta en un incremento de la vulnerabilidad para este sector. Otra consecuencia de la deforestación para el incremento del cambio climático está relacionada con el papel que juegan las plantas como sumideros fundamentales del carbono atmosférico, de tal suerte que al eliminarlos a través de la deforestación se incrementa el calentamiento global, dado que el $\mathrm{CO}_{2}$ es un gas de efecto invernadero (Gonzales Inca y Llanos López, 2015). En palabras de una persona entrevistada:

La deforestación empeora mucho la condición del campesino. Aleja la lluvia, calienta el suelo y hace que el calor empeore. Antes uno estaba en el campo trabajando y descansaba debajo de un árbol y la sombra era fresca y rica. Ahora casi ya no hay sombras para descansar porque nosotros mismos fuimos derrumbando todos nuestros árboles (testimonio entrevistado 10). 
En resumen, las personas que asocian las causas del cambio climático con el uso indiscriminado de agroquímicos apuntan que el cambio en la estacionalidad de las lluvias coincide con el periodo en el cual empezaron a sembrar el jitomate y otras hortalizas, hace alrededor de veinte años, las cuales demendan el uso de agroquímicos.

Para agregar a la reflexión sobre el binomio cambio climático -uso de agroquímicos- vale la pena indagar acerca de la contribución de los fertilizantes sintéticos en la emisión de los gases de efecto invernadero, que son finalmente los que impactan de manera decisiva en el incremento de la temperatura de la atmósfera y de los océanos. A nivel mundial, la agricultura industrial se caracteriza por el uso masivo de fertilizantes sintéticos nitrogenados y las emisiones resultantes de $\mathrm{N}_{2} \mathrm{O}$ representan el mayor porcentaje de contribución agraria al cambio climático; de hecho, la agricultura industrial es la principal actividad emisora de este contaminante. El óxido nitroso es el tercer gas de efecto invernadero en importancia con un potencial de calentamiento global alrededor de 300 veces superior al $\mathrm{CO}_{2}$ y una vida de 120 años, que representa cerca de $8 \%$ de las emisiones totales de gases de efecto invernadero. En este tenor, la fabricación de los fertilizantes nitrogenados demanda una gran cantidad de energía, de tal suerte que constituyen el insumo con mayor componente energético de la producción agrícola, lo cual supone 1\% del consumo energético mundial (Bermejo, 2010).

Frente a estos datos, que sin duda reflejan un punto de inflexión para la habitabilidad en el planeta en general y en México en particular, nos preguntamos si Sagarpa y Conagua no tendrían que implementar políticas agrícolas orientadas a mitigar estos efectos y por lo tanto transiten hacia el paradigma de la agroecología. ¿Por qué en la Meseta Comiteca las instituciones gubernamentales, tanto federales como estatales o municipales, siguen apostando a estrategias agrícolas altamente demandantes de fertilizantes nitrogenados químicos?

Los datos expuestos se suman al incremento de las condiciones de vulnerabilidad de los campesinos en la zona de estudio, la ausencia de instituciones públicas efectivas con una visión integral de sustentabilidad y, por ende, con programas de agroecología, manejo de cuencas, seguros agrícolas, reforestación, diversificación y adaptación productiva, entre otros (Deressa et al., 2011). Frente a la inercia institucional que no logra hacer una lectura dinámica de los nuevos retos que enfrentamos en el campo mexicano, la población rural tiene que recurrir principalmente a sus propios conocimientos, tecnologías y redes sociales. El desinterés de la parte gubernamental-local por el tema, traducido a falta de programas y proyectos concretos para la mitigación y adaptación al cambio climático, lleva consigo que la información científica cuantitativa y cualitativa no se baje a nivel de piso. Cobra entonces sentido la percepción y las acciones concretas de adaptación de parte de los campesinos en el plano local; por ejemplo, buscar dentro de su material genético local las variedades de maíz de ciclo corto (tres a tres meses y medio) para mitigar el retraso de la lluvia o su ausencia.

\section{IMPACTOS DEL CAMBio CLIMÁtico EN EL COTIDIANO DE LAS PERSONAS ENTREVISTADAS}

Las percepciones sobre los impactos del cambio climático se pueden agrupar en ámbitos culturales, económicos y sociales. Si bien se hace esta distinción en términos de apoyo para el análisis, es evidente que los ámbitos están relacionados y se configura una espiral viciosa, en donde el impacto cultural agrava el de orden económico y a su vez el económico incide en el social.

En términos culturales, el cambio en la estacionalidad de las lluvias ha provocado que un sistema tradicional milenario de cultivo de milpa, conocido como sistema pul-ha, esté en desuso. Pul-ha es una palabra tojolabal y quiere decir cubeta con agua ( $h a$ en tojolabal es agua y pul significa cubeta o balde). En este sistema se preparaba el terreno a finales de enero para sembrar en la primera quincena de febrero. Y se sembraba la milpa en febrero porque entre febrero y abril había por lo menos dos eventos fuertes de lluvias que ayudaban a mantener vivas las plantas pequeñas de maíz. En la actualidad ya no es seguro que llueva en estas fechas; en cambio, es más común que las lluvias empiecen entre junio y julio. 
En general hay un consenso entre las personas entrevistadas sobre la existencia de cambios considerables en la periodicidad de las lluvias. El conocimiento de la población campesina ubica el periodo de lluvias entre los meses de mayo a octubre, con fenómenos aislados en marzo, como una normalidad. Sin embargo, en los últimos años se observan cambios, puesto que se nota la ausencia en marzo y el inicio de la temporada muy retrasado, incluso llegar a julio sin lluvia. Es decir, un comportamiento totalmente atípico. La afectación por el retraso de las lluvias en estos meses es la pérdida de un ciclo agrícola de milpa tradicional (pul-ha).

Dado que el cultivo pul-ha demanda mucha mano de obra, esto también constituye un factor para que se vaya perdiendo de manera gradual dicha práctica cultural y productiva; no obstante, varios entrevistados afirman que el principal motivo por el cual ya no se animan a sembrar el pul-ha es el cambio en la estacionalidad de las lluvias, porque ya no asegura la sobrevivencia de las plantas.

El impacto económico del cambio climático en la región se manifiesta a través de la pérdida de las cosechas en consecuencia de un periodo de sequía de hace dos años. Los productores más descapitalizados tuvieron que dejar sus parcelas para trabajar como mano de obra en parcelas de agricultores con mayor poder adquisitivo que siembran hortalizas. De esa manera, el impacto económico ha ocasionado consecuencias productivas al reducir la superficie sembrada, y no se observa que las instituciones relacionadas con el desarrollo rural estén apoyando a los campesinos a través de programas para la recuperación productiva y de adaptación al cambio climático. A propósito, un testimonio:

El campesino sólo pierde. Pierde su dinero invertido en el campo porque no hay lluvia y las instituciones no apoyan con nada. Mucha gente está dejando de sembrar porque ya no les conviene. Es mucha perdedera de dinero. Algunos de aquí van a Comitán a trabajar en las parcelas de allá porque ya no pueden mantener a sus parcelas, porque es mucho riesgo con eso de que ya no sabemos cuándo vienen las lluvias (testimonio entrevistado 9).

Asimismo, el impacto económico de la pérdida de las cosechas por la sequía trajo como consecuencia para muchas familias la necesidad de migrar, como una estrategia para capitalizarse y así lograr pagar las deudas contraídas para el cultivo del tomate. La migración es pendular, dentro (Comitán y Tuxtla) o hacia fuera del estado, principalmente Quintana Roo (Playa del Carmen y Puerto Aventuras). También se identifica un sector de jóvenes que migra para los Estados Unidos de América.

\section{Conclusiones}

En la zona de estudio existe una opinión generalizada sobre el cambio del clima regional en los últimos años, la cual fue explicada por los actores sociales locales a través de la irregularidad de las lluvias, cambios en los periodos de granizadas e incremento del calor y la temperatura. Así pues, asocian estos cambios a factores simbólicos religiosos y antrópicos relacionándolos con el castigo divino, la deforestación y el uso indiscriminado de agroquímicos. De este modo, las manifestaciones del cambio climático en el imaginario local están muy cercanas a la cotidianidad campesina.

La gran mayoría de las personas entrevistadas no tiene un discurso teórico sobre el cambio climático; de hecho, manifestaron no conocer el término y sólo se pudo entrar en el debate acerca del tema a partir de la percepción de sus manifestaciones a nivel local. Dicha situación plantea un desafío para las políticas y programas de adaptación y mitigación del cambio climático, dado que para actuar sobre determinado problema con éxito es necesario que exista una percepción local de que el problema realmente está presente, con el fin de generar interés por adoptar cambios.

Por ser el cambio climático un problema aún en construcción a nivel comunitario, es necesario el trabajo de formación-capacitación-comunicación climática para crear conocimiento sobre el tema y formular propuestas e iniciativas e incorporarlas en las políticas locales. Es decir, que se construya una percepción pública del problema y sus consecuencias a nivel global y no se quede con la idea de que el problema es local 
y no tiene consecuencias desastrosas en el resto del mundo. Se requiere la generación de una fuerte demanda local por programas y acciones hacia el cambio climático.

A pesar de que gran parte de las personas entrevistadas haya tenido pérdidas de sus cosechas como consecuencia de los cambios en el clima, no se cuestionan sobre la necesidad de realizar alteraciones en sus sistemas productivos. Estos datos señalan que estos productores en particular presentan dificultades para enfrentar y adaptarse al cambio climático, entre otros motivos, debido a que les es un tema poco conocido por la carencia de información y recursos para implementar los cambios; no obstante los planteamientos de Hernández Xolocotzi (1998), quien asevera que 9000 años de agricultura campesina en México, con constantes cambios en el medio natural, hacen que los campesinos mexicanos estén en procesos de adaptación muchos años antes del concepto de cambio climático antropogénico.

Las instituciones gubernamentales relacionadas con políticas para el campo tienen el gran reto de innovar y generar alternativas de adaptación que sean cultural, ambiental y económicamente viables para los agricultores locales. También, las políticas en la materia tienen que crear sinergias con otras políticas del sector ambiental incluso con los sectores social y económico, así como con otros instrumentos de planificación. Por ello, es importante integrar las opciones y medidas de adaptación al cambio climático en otras políticas en curso. Es urgente el diseño e implementación de programas que tengan como objetivo informar, sensibilizar, capacitar y ofrecer oportunidades para que los agricultores incrementen su capacidad de adaptación al cambio climático.

\section{SUGERENCIAS Y PROSPECTIVA}

Investigaciones como la de este trabajo, centradas en la percepción social, permiten que se aporte una lectura social que delinee ejes de actuación para atender el cambio climático, dado que aceptar las políticas de adaptación por actores locales dependerá en gran medida de sus percepciones sobre la naturaleza, gravedad y posibles consecuencias del problema. Consideramos que el estudio de las percepciones ambientales puede ser una poderosa herramienta para ayudar a comprender la relación seres humanos-naturaleza, ya que está determinada por la forma en que se percibe el entorno. Las percepciones orientan las decisiones sobre las estrategias de manejo de los recursos y conocimiento de su base. Tomando en cuenta a diferentes actores sociales, contribuye al diseño de políticas públicas encaminadas a promover procesos de adaptación desde lo local.

En futuras investigaciones se sugiere hacer un análisis sobre el papel que juega el estudio de percepciones de las poblaciones locales en la comprensión de sus comportamientos, así como su relación con el diseño de políticas públicas de atención al cambio climático. A partir de la percepción se pueden sistematizar los procesos de adaptación al cambio climático por parte de agricultores en diferentes regiones de la República Mexicana, dado que la experiencia que ya existe en las comunidades campesinas sobre el cambio climático y sus impactos debería constituirse un punto de partida para el diseño de políticas públicas de adaptación. Es imprescindible realizar estudios en diferentes zonas del país con el objetivo de rescatar el conocimiento campesino sobre el clima y sistematizar sus estrategias de adaptación para tomarlas como base de propuestas de políticas.

En este tenor, se hace necesario conocer y comprender los escenarios de cambio climático para la región, así como profundizar en el análisis de las alternativas productivas de adaptación para la zona. Con esto, se hace urgente la generación de estudios cualitativos y cuantitativos que recuperen los métodos utilizados por los agricultores para adaptarse al cambio climático por medio de un análisis de los factores que influyen en sus selecciones y las barreras que enfrentan para lograr adaptarse. Según argumenta Berkes et al. (2000), los conocimientos y experiencias de las comunidades rurales son complementarios al conocimiento científico y las propuestas de desarrollo local elaboradas desde este paradigma de complementariedad entre saberes tienen mucho más oportunidad de lograr que se impulsen procesos de sustentabilidad. 


\section{Agradecimientos}

Investigación realizada en el marco del proyecto "Impactos socioambientales del cambio climático registrados en la cuenca del Río Conchos y del Río Usumacinta, de acuerdo con criterios del IPCC 2014”, financiado por el Fondo Sectorial Semarnat-Conacyt.

\section{REFERENCIAS}

Berkes, F., Colding, J. y Folke, C. (2000). Rediscovery of traditional ecological knowledge as adaptive management. Ecological Applications, 10(5), 1251-1262.

Bermejo, I. (2010). El agrícola es el sector con más emisiones de efecto invernadero a escala mundial. Área de Agroecología de Ecologistas en Acción. Revista El Ecologista, 67. Disponible en http:// www.ecologistasenaccion.org/article19945.html

Deressa, T. T., Hassan, R. M. y Ringler, C. (2011). Perception of and adaptation to climate change by farmers in the Nile basin of Ethiopia. Journal of Agricultural Science, 149(1), 23-31.

Flores Moreno, A. y Valdivia Corrales, G. (2011). Las percepciones de la población rural campesina de la Microcuenca Mollebamba sobre la incidencia del cambio climático en su forma de vida. Serie Investigación 5. Microcuenca Mollebamba. Cusco: Centro Bartolomé de las Casas, PACC Perú, Agencia Suiza para el Desarrollo y la Cooperación.

García García, A., Zárate Toledo, M. A., Valencia Barrera, E. y Sánchez Sánchez, J. (2015). Diagnóstico: "transferencia de tecnología para el manejo y conservación del suelo y agua en el Distrito de Temporal Tecnificado 011 MargaritasComitán; Estado de Chiapas". Comitán: Conagua, Organismo de Cuenca Frontera Sur.

Giddens, A. y Sutton, P. (2013). Sociology. London: Polity Press.

Gonzales Inca, C. A. y Llanos López, R. (2015). Reporte Técnico Evaluación de los efectos de la deforestación en la hidrología y pérdida lateral de carbono orgánico del suelo de la cuenca del Alto Mayo. Lima: Conservación Internacional Perú.

Harris, M. (1982). El materialismo cultural. Madrid: Alianza Editorial.

Hernández Xolocotzi, E. (1998). Agricultura Campesina ¿Obstáculo o alternativa?, en M. A. Díaz León y A. Cruz León (comps.), Nueve mil años de agricultura en México, Homenaje a Efraím Hernández Xolocotzi (pp. 132-136). México: Universidad Autónoma Chapingo.

Hogan, D. J. (2007). Human dimensions of global environmental change. Ambiente \& Sociedade, 10, 161-166.

INEGI (Instituto Nacional de Estadística y Geografía). (2011). XII Censo General de Población y Vivienda 2010.

IPCC (Intergovernmental Panel on Climate Change). (2014). Cambio climático 2014. Informe de sintesis. Contribución de los Grupos de trabajo I, II y III al Quinto Informe de Evaluación del Grupo Intergubernamental de Expertos sobre el Cambio Climático. Ginebra: IPCC.

IPCC (Intergovernmental Panel on Climate Change). (2008). Cambio climático 2007. Informe de sintesis. Ginebra: IPCC.

Kuhnem, A. e Higuchi, M. I. G. (2011). Percepção ambiental, en S. Cavalcante y G. A. Elali (coord.), Temas básicos em Psicología Ambiental (pp. 28-52). Petrópolis: Vozes.

Maddison, D. J. (2007). The Perception of and Adaptation to Climate Change in Africa. Disponible en http:// papers.ssrn. com/sol3/papers.cfm?abstract_id $=1005547$.

Mariño, N. (2011). Reflexiones sobre la perspectiva cultural en las políticas de cambio climático en Colombia: un acercamiento al análisis cultural y espacial de las políticas públicas, en A. Ulloa (ed.), Perspectivas culturales del clima (pp. 495-528). Bogotá: Universidad Nacional de Colombia. Biblioteca abierta: Perspectivas Ambientales.

Marson Teixeira de Andrade, R. y Miccolis, A. (2012). Diagnóstico de percepção de risco ambiental e mudança climática no Núcleo Rural da Microbacia do Córrego do Urubu. Ponencia presentada en VI Encontro Nacional da anppas. Belém, Brasil. 
Paes de Menezes, L. C., Carneval de Oliveira, B. M.y Giovanetti El-Deir, S. (2011). Percep̧̧ão ambiental sobre mudanças climáticas: estudo de caso no semiárido pernambucano. Ponencia presentada en II Congresso Brasileiro de Gestão Ambiental, Londrina, Paraná. Disponible en http://www.ibeas.org.br/congresso2/

Pike, K. L. y Pike, E. G. (1991). El análisis gramatical. México: Universidad Nacional Autónoma de México.

Pinilla-Herrera, M. C., Rueda, A., Pinzón, C. y Sánchez, J. (2012). Percepciones sobre los fenómenos de variabilidad climática y cambio climático entre campesinos del centro de Santander, Colombia. Ambiente y Desarrollo, 16 (31), 25-37.

PNUD (Programa de las Naciones Unidas para el Desarrollo). (2008). Guía recursos de género para el cambio climático. Ciudad de México: PNUD.

Rossbach de Olmos, L. (2011). Del monólogo científico a las pluralidades culturales: dimensiones y contextos del cambio climático desde una perspectiva antropológica, en A. Ulloa (ed.), Perspectivas culturales del clima (pp. 34-55). Bogotá: Universidad Nacional de Colombia.

Slovic, P. (1987). Perception of risk. Science. New Series, 236(4799), 280-285.

Soares, D. y García, A. (2014). Percepciones campesinas indígenas acerca del cambio climático en la cuenca de Jovel, Chiapas-México. Cuadernos de Antropología Social, 39, 63-89.

Soares, D. y Romero, R. (2008). Recursos hídricos en la cuenca del Amacuzac. Inventio, 4(8), 13-23.

Tuan, Y. (1980). Topofilia: um estudo da percepção, atitudes e valores do meio ambiente. São Paulo: Difel.

VanderMolen, K. (2011). Percepciones de cambio climático y estrategias de adaptación en las comunidades agrícolas de Cotacachi. Ecuador Debate, 82, 145-158.

Whyte, A. V. T. (1977). Guidelines for fields studies in Environmental Perception. Paris: Unesco/MAB.

\section{Notas}

[1] El estado de Chiapas está dividido en 15 regiones económicas. La Región XV Meseta Comiteca Tojolabal comprende los municipios de Comitán de Domínguez, La Independencia, Las Margaritas, Las Rosas, La Trinitaria, Maravilla Tenejapa y Tzimol.

[2] El uso de agroquímicos se incrementó indiscriminadamente con el cultivo de tomate. Por ejemplo, para el control de la mosca blanca se utilizan hasta doce aplicaciones cuando el máximo es de cuatro.

\section{BY-NC-ND}

